\section{Targeting germ-cell adhesion in the pursuit of a male contraceptive}

In a new study, Mruk and colleagues demonstrate a novel method whereby a mutant folliclestimulating hormone ( $\mathrm{FSH}$ ) protein acts as a carrier for adjudin to induce epithelial germ-cell loss in the testis and, consequently, infertility in adult male rats. Previous studies have shown oral administration of large doses of adjudin induces germ-cell loss, but with severe adverse effects in a small subset of animals. adjudin alone is unsafe because of its subchronic toxicity; however, conjugating it to a carrier such as FSH improves its efficacy and selectivity and reduces its toxicity.

To assess the efficacy and reversibility of treatment with an adjudin-FSH-mutant conjugate, the authors administered the conjugate $(50 \mu \mathrm{g} / \mathrm{kg})$ intraperitoneally to rats $(n=8)$. Control animals received either native rat $\mathrm{FSH}$ or the FSH mutant alone. Intraperitoneal administration of adjudin $0.5 \mu \mathrm{g} / \mathrm{kg}$ resulted in infertility, and similar results were obtained when $50 \mathrm{mg} / \mathrm{kg}$ was administered orally. After 4 weeks of treatment with the conjugate, more than $50 \%$ of spermatocytes were depleted, and after 6 weeks spermatocytes were found in $\sim 30 \%$ of examined tubules.

The authors conclude that the efficacy and safety of the adjudin-FSH-mutant conjugate delivered to the testis via parenteral administration is substantially greater than that of orally administered adjudin. The authors suggest that this compound could become a male contraceptive for human use.

Original article Mruk DD et al. (2006) A male contraceptive targeting germ cell adhesion. Nat Med 12: 1323-1328

\section{Long-term outcomes of endopyelotomy and pyeloplasty are worse than expected}

Ureteropelvic junction obstruction has traditionally been treated with open pyeloplasty, but antegrade endopyelotomy is a less-invasive, widely accepted, alternative therapy. Dimarco and colleagues have compared the long-term outcomes of these two treatments.

The authors retrospectively reviewed the medical records of patients with a complete history who underwent either endopyelotomy ( $n=182$, mean age 47.2 years) or pyeloplasty ( $n=175$, mean age 42.8 years) at a single center.

Symptoms of obstruction resolved completely and radiographic improvements were observed in $61.0 \%$ of patients who underwent endopyelotomy and in $82.3 \%$ of patients who underwent pyeloplasty. The 3, 5, and 10 year rates of recurrence-free survival were $63 \%$, $55 \%$, and $41 \%$ for endopyelotomy patients and $85 \%, 80 \%$, and $75 \%$ for pyeloplasty patients, respectively. Treatment failure rates after 1 year were $46.5 \%$ for endopyelotomy and $42.2 \%$ for pyeloplasty, both worse than were expected. Previous pyeloplasty adversely affected the success of salvage pyeloplasty $(P=0.009)$ but not that of salvage endopyelotomy. Previous endopyelotomy, however, did not affect the success of subsequent endopyelotomy or pyeloplasty. At pyeloplasty, the authors found that $38 \%$ of patients had crossed vessels, but this fact did not account for the high failure rate of endopyelotomy, because only $31 \%$ of patients who underwent salvage pyeloplasty after failed endopyelotomy had crossed vessels.

The gap in success rates between pyeloplasty and endopyelotomy continues to broaden with extended follow-up, and a substantial number of treatment failures occur after 5-10 years. The authors stress the need for follow-up imaging, and question whether endopyelotomy should be offered as a primary treatment for ureteropelvic junction obstruction.

Original article Dimarco DS et al. (2006) Long-term success of antegrade endopyelotomy compared with pyeloplasty at a single institution. J Endourol 20: 707-712

\section{A new $\alpha_{1 A}$-adrenoceptor- selective antagonist for treating benign prostatic hyperplasia}

At present, $\alpha_{1}$-adrenoceptor antagonists such as tamsulosin are widely used in first-line treatment for benign prostatic hyperplasia (BPH) associated with lower urinary tract symptoms (LUTS). Kawabe et al. carried out a study comparing the efficacy and safety of silodosin, a new, highly selective $\alpha_{1 A}$-adrenoceptor antagonist, with that of tamsulosin in patients with LUTS associated with BPH. 